\title{
Visual Histological Grading System for the Evaluation of in Vitro-Generated Neocartilage ${ }^{\dagger}$
}

\author{
SHAWN PATRICK GROGAN, Ph.D., ${ }^{1, *}$ ANDREA BARBERO, Ph.D., ${ }^{2}$ VERENA \\ WINKELMANN, H.F.P., ${ }^{1}$ FRANZ RIESER, B.Sc., ${ }^{3}$ JAMES S. FITZSIMMONS, B.Sc., ${ }^{4}$ SHAWN \\ O'DRISCOLL, M.D., Ph.D., ${ }^{4}$ IVAN MARTIN, Ph.D., ${ }^{2}$ and PIERRE MAINIL-VARLET, M.D., Ph.D. ${ }^{1}$
}

\begin{abstract}
Here we present the development of a visual evaluation system for routine assessment of in vitro-engineered cartilaginous tissue. Neocartilage was produced by culturing human articular chondrocytes in pellet culture systems or in a scaffold-free bioreactor system. All engineered tissues were embedded in paraffin and were sectioned and stained with Safranin $\mathrm{O}$-fast green. The evaluation of each sample was broken into 3 categories (uniformity and intensity of Safranin $O$ stain, distance between cells/amount of matrix produced, and cell morphology), and each category had 4 components with a score ranging from 0 to 3. Three observers evaluated each sample, and the new system was independently tested against an objective computer-based histomorphometry system. Pellets were also assessed biochemically for glycosaminoglycan (GAG) content. Pellet histology scores correlated significantly with GAG contents and were in agreement with the computer-based histomorphometry system. This system allows a valid and rapid assessment of in vitro-generated cartilaginous tissue that has a relevant association with objective parameters indicative of cartilage quality.
\end{abstract}

\section{INTRODUCTION}

A RTICULAR CARTILAGE REPAIR using cell-based methods has been the subject of much interest, with autologous chondrocyte implantation (ACI), the leading clinical strategy in humans. ${ }^{1-3}$ The increased number of published works in various animal models over the past 10 years reflects efforts to prefabricate implantable neocartilage. $^{4-10}$ With such activity, means to assess the repair success using histological grading systems have been developed. ${ }^{11-13}$

Although these accepted grading systems have been used extensively for the assessment of cartilage repair, they are not readily applicable to the assessment of tissue-engineered cartilage and pellet cultures. Many research laboratories conducting basic studies of chondrogenesis using chondrocytes or mesenchymal stem cells employ pellet cultures, especially where human cells are in rare supply or multiple variables with expensive growth factors need to be screened. ${ }^{14-18}$

Assessing the quality of tissue-engineered constructs is routinely conducted using a range of techniques. Histological analysis is essential to assess the structure of the neo-tissue and to visualize the extent and components of extracellular matrix produced..$^{19}$ Immunohistochemical, biochemical, biomechanical, and specific gene-expression studies are also

\footnotetext{
${ }^{1}$ Osteoarticular Research Group, Institute of Pathology, University of Bern, Bern, Switzerland.

${ }^{2}$ Research Division of the Department of Surgery, University of Basel, Basel, Switzerland.

${ }^{3}$ Zimmer Orthopedics, Winterthur, Switzerland.

${ }^{4}$ Cartilage and Connective Tissue Research, Mayo Clinic College of Medicine, Rochester, Minnesota.

*Present address of Shawn Patrick Grogan is The Division of Arthritis Research, The Scripps Research Institute, La Jolla, California.

${ }^{\dagger}$ This work was presented as an abstract at the International Cartilage Repair Society 2004 meeting in Ghent, Belgium, with the same title (poster presentation). Experimental work was performed at the University of Basel and Bern.
} 
employed to augment these assessments. The 2 most definable elements used to study articular cartilage consist of the presence of collagen type II and glycosaminoglycans (GAGs). Although collagen type II has been used to assess the differentiation state of the resident chondrocytes, ${ }^{20}$ GAG has been used frequently to assess many aspects of cartilage status, including cartilage degradation, ${ }^{21-23}$ cartilage quality, ${ }^{1-13}$ and chondrogenic capacity, ${ }^{24}$ and as a guide to extracellular matrix maturity. ${ }^{5}$

Safranin O intensity positively correlates with the GAG content measured using biochemical analysis of ${ }^{35} \mathrm{~S}$-labeled cartilage or fixed charge density. ${ }^{25-27}$ Consequently, the staining intensity of Safranin $\mathrm{O}$ has been used in many computerized image analyses of cartilage $e^{28-31}$ and in vitrocultivated tissue. ${ }^{32,33}$ Indeed, Martin et al. ${ }^{32}$ measured the intensity and the spatial distribution of red in Safranin Ostained sections of engineered cartilage. The intensity of stain was found to be in proportion to the wet weight fraction of GAG.

O'Driscoll et $a .^{33}$ tested the validity of a subjective histological-histochemical scoring system based on the visual estimation of percentage of cartilage alone, and compared it with a previously developed objective automated histomorphometry program. ${ }^{34}$ Although the objective system in this study was preferred, the subjective grading of the percentage cartilage was deemed valid. This system, however, does not account for other features frequently seen in engineered tissue, such as different cell morphologies present and the non-uniformity or heterogeneity of the matrix products represented. Assessment of such phenomenon may be of interest for fine-tuning techniques (e.g., cell culture environments) and may also influence the success of engineered tissue once implanted into cartilage defects.

In this current study, we aimed to develop a visual scoring system (Bern Score) that may be used as a reference for researchers to grade cartilaginous pellet cultures and engineered tissue. We also developed the scoring system to take into account features such as cell morphology and construct heterogeneity. To validate this system, we tested the graded tissue against a biochemical index (GAG content) and the computer-based histomorphometric system described by O'Driscoll et al. ${ }^{34}$

\section{MATERIALS AND METHODS}

\section{Chondrocyte isolation and expansion}

Human articular cartilage was obtained from 40 cadavers under local ethical approval within $24 \mathrm{~h}$ post mortem. Chondrocytes were released via enzymatic digestion with $0.15 \%$ type II collagenase for $22 \mathrm{~h}$ and expanded for 2 passages. Cells for pellet cultures were expanded in Dulbecco's modified Eagle medium (DMEM) with 10\% fetal calf serum, supplemented with transforming growth factor $\beta 1$, fibroblast growth factor 2 , and platelet-derived growth factor bb for approximately 2 weeks (see Barbero et $a l .{ }^{24}$ for details). Only DMEM with $10 \%$ fetal calf serum was used to expand cells for seeding into the scaffoldfree bioreactor system. ${ }^{35}$

Pellet cultures. Post-expanded chondrocytes were placed in pellet cultures using a defined serum-free medium, as previously described. ${ }^{24}$ In summary, cells were suspended in DMEM supplemented with ITS $^{+1}$ (Sigma Chemical, St. Louis, MO), $10^{-7} \mathrm{M}$ dexamethasone (Sigma), and $10 \mathrm{ng} / \mathrm{mL}$ transforming growth factor $\beta 1$ (R\&D Systems, Minneapolis, $\mathrm{MN})$. Aliquots of $5 \times 10^{5}$ cells $/ 0.5 \mathrm{~mL}$ were centrifuged at $1200 \mathrm{rpm}$ for $5 \mathrm{~min}$ in $1.5 \mathrm{~mL}$ polypropylene conical tubes (Sarstedt, Nümbrecht, Germany) to form spherical pellets, which were placed onto a 3-dimensional orbital shaker (Bioblock Scientific, Frenkendorf, Switzerland) at $30 \mathrm{rpm}$. Pellets were cultured for 2 weeks, with medium changes twice per week, and assessed histologically (Safranin O-fast green staining) and biochemically (GAG content). Typically, 2 pellets were used for each assay for direct comparison between these 2 parameters.

Biochemical Evaluation. For the assessment of total GAG content of pellet cultures, each sample was first digested with protease $\mathrm{K}(0.5 \mathrm{~mL}$ of $1 \mathrm{mg} / \mathrm{mL}$ protease $\mathrm{K}$ in $50 \mathrm{mM}$ Tris with $1 \mathrm{mM}$ ethylenediaminetetraacetic acid, $1 \mathrm{mM}$ iodoacetamide, and $10 \mu \mathrm{g} / \mathrm{mL}$ pepstatin-A for $15 \mathrm{~h}$ at $\left.56^{\circ} \mathrm{C}\right){ }^{36}$ GAG quantity was measured spectrophotometrically using dimethylmethylene blue, ${ }^{37}$ with chondroitin sulfate as a standard, and normalized to the deoxyribonucleic acid (DNA) amounts, measured spectrofluorometrically using the CyQUANT Kit (Molecular Probes, Eugene, OR) and with calf thymus DNA as a standard. GAG contents are reported as $\mu \mathrm{gGAG} / \mu \mathrm{gDNA}$.

Production of engineered neocartilage. Post-expanded chondrocytes were cultured in a scaffold-free bioreactor system as previously described for porcine chondrocytes. ${ }^{35}$ Briefly, $8 \times 10^{6}$ cells were seeded into bioreactor chambers and maintained within the reactor for 3 weeks in the same media used for expansion, with media changes every 3 to 4 days. Subsequently, the formed cartilaginous tissues were removed and placed in culture media, each in 1 well of a 6well plate, and further cultured for 1 week. After cultivation time, the neocartilage was fixed in $4 \%$ paraformaldehyde and embedded in paraffin for archive storage. For the current study, the paraffin blocks were retrieved for staining with Safranin O-fast green.

\section{Safranin O-fast green staining}

Paraffin-embedded tissues (pellet and engineered) were sliced at 3 to $4 \mu \mathrm{m}$ and mounted on glass slides (Super Frost color, Menzel-Gläser, Freiburg, Germany). The paraffin slices were immersed in xylene, twice for $5 \mathrm{~min}$, to remove paraffin and then re-hydrated in an alcohol series (twice 
$100 \%, 95 \%$, and $70 \% ; 2$ min each) and finally in distilled water for $5 \mathrm{~min}$. For Safranin O staining $(0.2 \mathrm{~g}$ Safranin T, Fluka, Buchs, Switzerland; $1 \mathrm{~mL} 100 \%$ acetic acid; $100 \mathrm{~mL}$ distilled water), the slides were immersed in the Safranin solution for $10 \mathrm{~min}$ followed by rinsing in distilled water. The sections were then further stained in fast green solution $(0.04 \mathrm{~g}$ fast green chroma; $0.2 \mathrm{~mL} 100 \%$ acetic acid; $100 \mathrm{~mL}$ distilled water) for $15 \mathrm{~s}$, rinsed in distilled water, and dried between filter paper. Subsequently, the slides were rinsed in absolute alcohol until excess fast green was removed, whereupon the Safranin O staining becomes apparent. After 2 washes in xylene (5 min each), a cover slip with Eukitt was placed over the stained tissue. The quality of Safranin O-fast green staining is routinely monitored with reference tissues of osteoarthritis (OA), normal, and growth-plate cartilage to reduce variations in staining intensities. The reagents used in this staining procedure must also be changed regularly (every 50 to 100 slides).

\section{Histology grading}

Examination. Each observer evaluated pellet culture $(n=25)$ and tissue-engineered sections $(n=20)$ stained with Safranin O-fast green under a light microscope (Axioskop, Zeiss, Germany) using the Bern Score system as described below. Each observer (1 pathologist and 2 basic scientists, experienced in the examination of engineered cartilage) graded each sample twice (pellet cultures and tissue-engineered cartilage) with an interval of 3 months between evaluations.

Grading Safranin $O$-fast green stained slides using the Bern Score. The Bern Score accounts for uniformity and intensity of matrix staining, cell density/extent of matrix produced, and cellular morphologies. Table 1 details the 3 criteria, each with equal weight, with a possible minimum collective score of 0 and a maximum of 9 . Sections were scored 1 category at a time and added to give a total score.

Category A: Uniformity and darkness of Safranin O-fast green stain. Each sample was observed with a $10 \times$ objective.

Category B: Distance between cells/amount of matrix accumulated was assessed with a $20 \times$ objective. Most neocartilage material is more highly cellular than native cartilage, but it is possible to distinguish differences in cellular distributions in pellet and tissue-engineered cartilage.

Category C: Cellular morphologies were viewed using a $40 \times$ objective. Based on the general consensus that a rounded cellular morphology is indicative of a chondrocyte phenotype, the presence of pyknotic/necrotic or fibroblast morphologies was interpreted to represent a negative quality and was scored accordingly.

The categories chosen in the Bern Score are likely to be interrelated. For instance, more-fibroblastic cells typically deposit matrix weakly stained for Safranin O.
Table 1. The Bern Score: For the Evaluation of Safranin O-Fast Green Stained Cartilaginous Pellet Cultures and Engineered Tissue Sections (Minimum Score: 0; MAXimum Score: 9)

Scoring categories Score

A. Uniformity and darkness* of Safranin O-fast green stain

No stain 0

Weak staining of poorly formed matrix

Moderately even staining

Even dark stain

B. Distance between cells/amount of matrix accumulated

High cell densities with no matrix in between (no spacing between cells)

High cell densities with little matrix in between (cells $<1$ cell-size apart)

Moderate cell density with matrix (cells approx. 1 cell-size apart)

Low cell density with moderate distance between cells $(>1$ cell) and an extensive matrix

C. Cell morphologies represented

Condensed/necrotic/pycnotic bodies

Spindle/fibrous

Mixed spindle/fibrous with rounded chondrogenic morphology

Majority rounded/chondrogenic

*Section 3-4 $\mu \mathrm{m}$ thick.

\section{Computer histomorphometric analysis}

Pellet culture $(n=25)$ and tissue-engineered sections $(n=20)$ that were graded using the Bern Score were also graded blindly using computerized histomorphometry to determine what proportion of the tissue produced was cartilage (percentage cartilage yield). These evaluations were performed with a custom-designed software application using the Vidas 2.1 Image Analysis System (Kontron customized by Carl Zeiss Canada, Don Mills, Ontario, Canada), which was previously validated for automated cartilage histomorphometry on periosteal explants. ${ }^{33}$ The analysis of cartilage is conducted automatically without the need of any manual "tracing" and is based on the uptake of Safranin $\mathrm{O}$ stain, with the thresholds and combinations of red, green, and blue distinguished automatically. The software algorithm discriminated each region (cartilaginous and noncartilaginous) based on its color.

\section{Photomicrographs}

Images of Safranin O-fast green stained sections of pellet cultures and engineered tissues used for the visual scales were captured using a microscope (Axiophot2, Ziess, Germany) fitted with a digital camera (Axiocam, Ziess, Germany) using $5 \times, 10 \times, 20 \times$, and $40 \times$ objectives. 


\section{Statistical analysis}

To test for correlations between the Bern Score and the computer-based histomorphometric system or between the Bern Score and the GAG content of pellet cultures, regression analysis was performed using Microsoft Excel97 (Microsoft Corp., Redmond, WA). Intra- and interobserver reproducibility was calculated as described by Moojen $e t a^{38}{ }^{38}$ The intra-observer reliability was generated from the difference of the 2 scores from a given observer and the mean of that section. Inter-observer reliability was derived from the difference between observer scores for a specific section and the average for that tissue sample.

\section{RESULTS}

From the pellet culture samples and engineered tissue constructs processed in this study, and from other archival material, we identified a variety of histological images (Fig. 1) that span the range of qualities normally produced in our hands using the 2 culture systems described in this study. Figure 1 represents images of Safranin O-fast green pellet cultures and tissue-engineered cartilage, from the generated paraffin block archive, that may be used as a visual reference for other researchers who are also interested in assessing the quality of neocartilage produced in their systems. Table 2 provides an overview of scores for each category to further illustrate the use of the Bern Score. As a convention, in situations in which a score in a particular category may be thought to be between the criteria outlined by the Bern Score, the grader should choose the upper score. A group consensus session after individual scoring may also be effective in adjudicating some difficult-tograde sections, especially those with multiple regions.

Representative examples of necrotic/pyknotic and fibroblast cellular morphologies, particularly observed in pellet
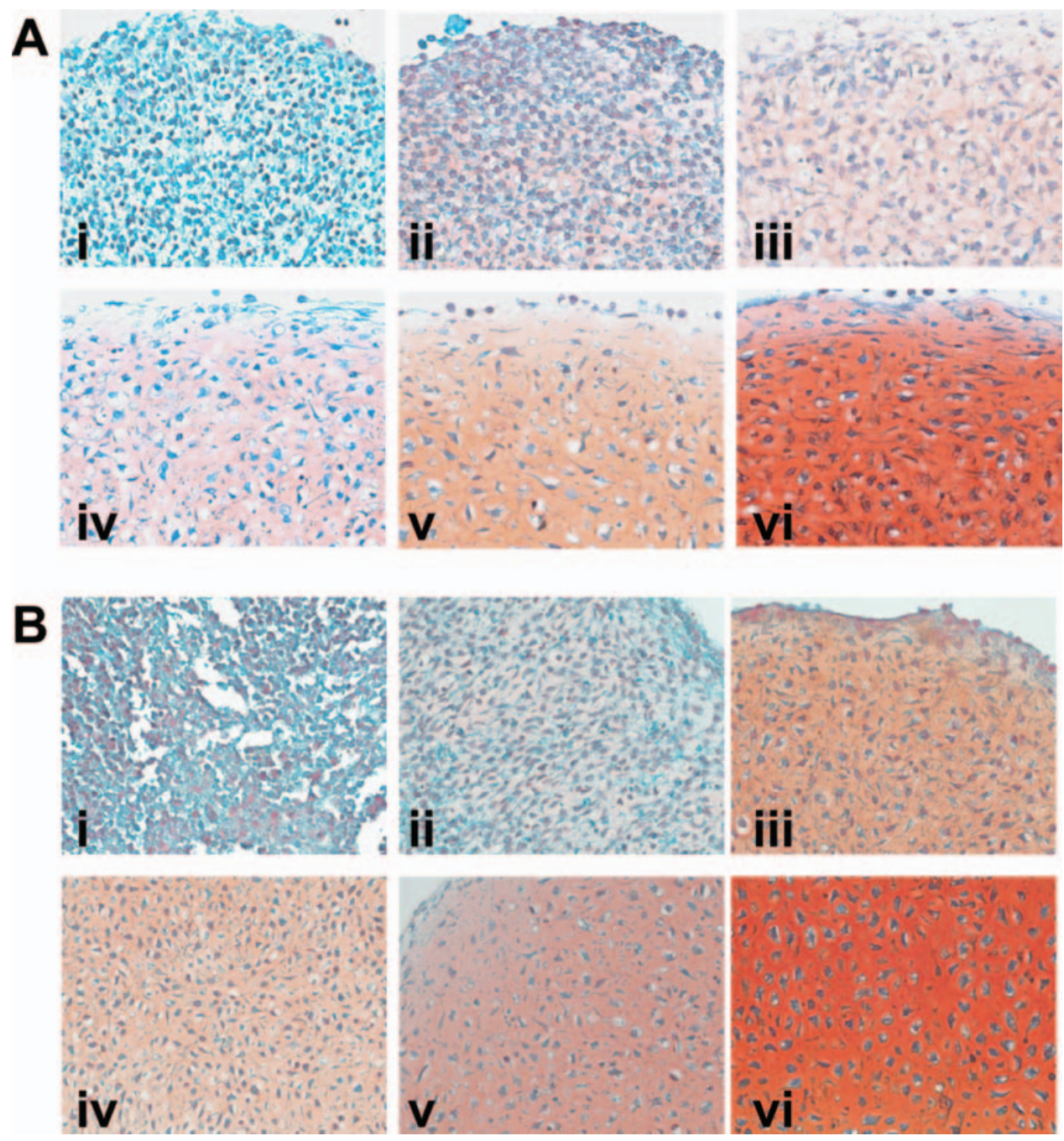

FIG. 1. Representative Safranin O-fast green images of (A) pellet cultures and (B) engineered cartilaginous tissue as a visual guide to scoring such cartilaginous tissues using the Bern Score provided in Table 1. The breakdown of these scores using the Bern Score is presented in Table 2 (magnification $40 \times$ ). Color images available online at www.liebertpub.com/ten. 
Table 2. Breakdown of Grades of Pellet and Engineered Neocartilage Represented in Fig. 1 Using the Bern Score (TABLE 1)

\begin{tabular}{lcccc}
\hline & \multicolumn{3}{c}{ Scoring category } \\
\cline { 2 - 4 } Image from & A: & B: Cell & C: & Total \\
Fig. 1 & Safranin $O$ & distance & Morphology & score \\
\hline Pellet (Ai) & 0 & 0 & 0 & $\mathbf{0}$ \\
Engineered (Bi) & 0 & 0 & 0 & $\mathbf{0}$ \\
Pellet (Aii) & 1 & 1 & 0 & $\mathbf{2}$ \\
Engineered (Bii) & 1 & 1 & 1 & $\mathbf{3}$ \\
Pellet (Aii) & 1 & 2 & 2 & $\mathbf{5}$ \\
Engineered (Biii) & 2 & 2 & 2 & $\mathbf{6}$ \\
Pellet (Aiv) & 2 & 2 & 2 & $\mathbf{6}$ \\
Engineered (Biv) & 2 & 2 & 3 & $\mathbf{7}$ \\
Pellet (Av) & 2 & 3 & 2 & $\mathbf{7}$ \\
Engineered (Bv) & 3 & 3 & 2 & $\mathbf{8}$ \\
Pellet (Avi) & 3 & 3 & 2 & $\mathbf{8}$ \\
Engineered (Bvi) & 3 & 3 & 3 & $\mathbf{9}$ \\
\hline
\end{tabular}

cultures, are depicted in Fig. 2. Most neocartilage formed in this study was homogenous with respect to the categories used in the Bern Score. However, in some constructs there was a heterogenous mixture of staining intensities and cell morphologies represented. An example of this phenomenon is illustrated in Fig. 3. In this field, there are at least 4 regions represented, and depending on the location, a score ranging from 4 to 9 may be given. To overcome this dilemma, we propose to identify each region, estimate the area it represents (as a percentage of the total area), and score each region individually. Each individual score is then adjusted with respect to the percentage area represented, and all scores are added together (100\%) to provide an overall single score (see example in Fig. 3).

A
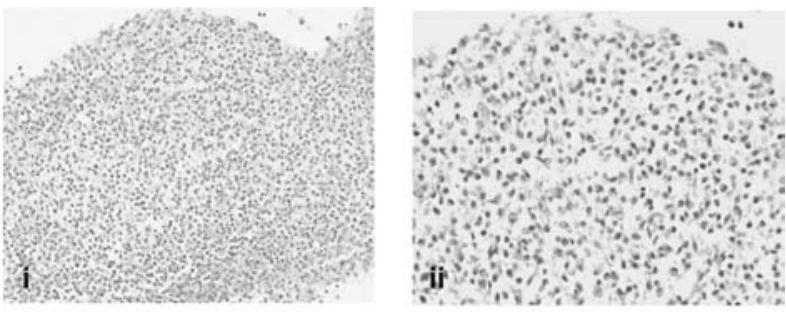

B
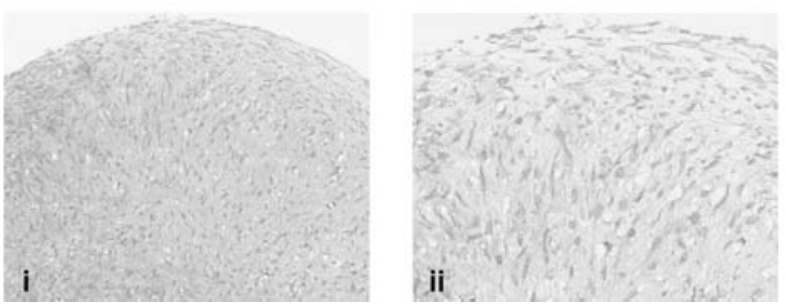

FIG. 2. Examples of pyknotic/necrotic (A) and fibrous type (B) morphologies found in engineered cartilage tissues (magnifications: $\mathrm{i}=10 \times$; $\mathrm{ii}=40 \times$ ).
A significant correlation between the Bern Score and the computer-based histomorphometric system was established for pellet cultures $\left(r^{2}=0.63 ; p<0.001 ; n=25\right)$ and tissueengineered neocartilages $\left(r^{2}=0.69 ; p<0.001 ; n=20\right)$. Pellet and tissue-engineered cartilage scores are presented in Fig. 4A $\left(r^{2}=0.68 ; p<0.0001 ; n=45\right)$. The Bern Scores were also found to significantly correlate $\left(r^{2}=0.78\right.$; $p<0.001 ; n=40$ ) with the biochemical attributes (GAG content) of pellet cultures (Fig. 4B). The intra-observer and inter-observer reproducibility is summarized in Table 3 . The mean overall intra-observer and inter-observer reproducibility were $-0.06 \pm 1.05$ and $-0.06 \pm 0.17$, respectively.

\section{DISCUSSION}

We have developed a visual histological grading system for the assessment of engineered cartilaginous tissues that correlates positively with computerized histomorphometry and biochemical measurements. The previously reported relationship between GAG content and pro-collagen type II gene expression ${ }^{24}$ further validates the described system.

The Bern Score is unique in that it not only grades cartilage per se, but also gives weight to non-cartilage-like areas that may be of interest to some investigators and prove important for the prediction of implant success. Recording and assessing the quality of neocartilage in this manner may be a basis to determine the effect of in vitro culture techniques and supplements on the development of cartilaginous tissues. The users of this system should define clearly what information is needed and compatible with an end goal. For instance, averaging scores for a general account of chondrogenesis may be compatible with adjusting culture conditions but not when grading engineered implants in which the presence of any necrotic tissue is not considered acceptable. The purpose of the images represented in this manuscript (Figs. 1 and 2) may aid other researchers in cartilage tissue engineering who wish to assess the quality of their engineered tissue. Additionally, we hope that the Bern Score will, at the very least, stimulate discussion in reaching a consensus or lead toward the development of a common language and system to evaluate such in vitro-generated tissues.

For the assessment of heterogeneous samples (i.e., sections with multiple cellular morphologies), we suggested a re-weighting approach that requires the scorer to estimate the percentage area represented by each region. In most instances, we found this method to be relatively straightforward, but in cases in which it may be difficult to discern percentage areas, we recommend group consensus scoring on those samples or on samples that show large interindividual variation. It may also be beneficial to have at least one trained pathologist to assist in such consensus scoring because this is a standard practice in pathology.

Other criteria commonly recognized as characteristic of cartilage might have been chosen in this system. Collagen 

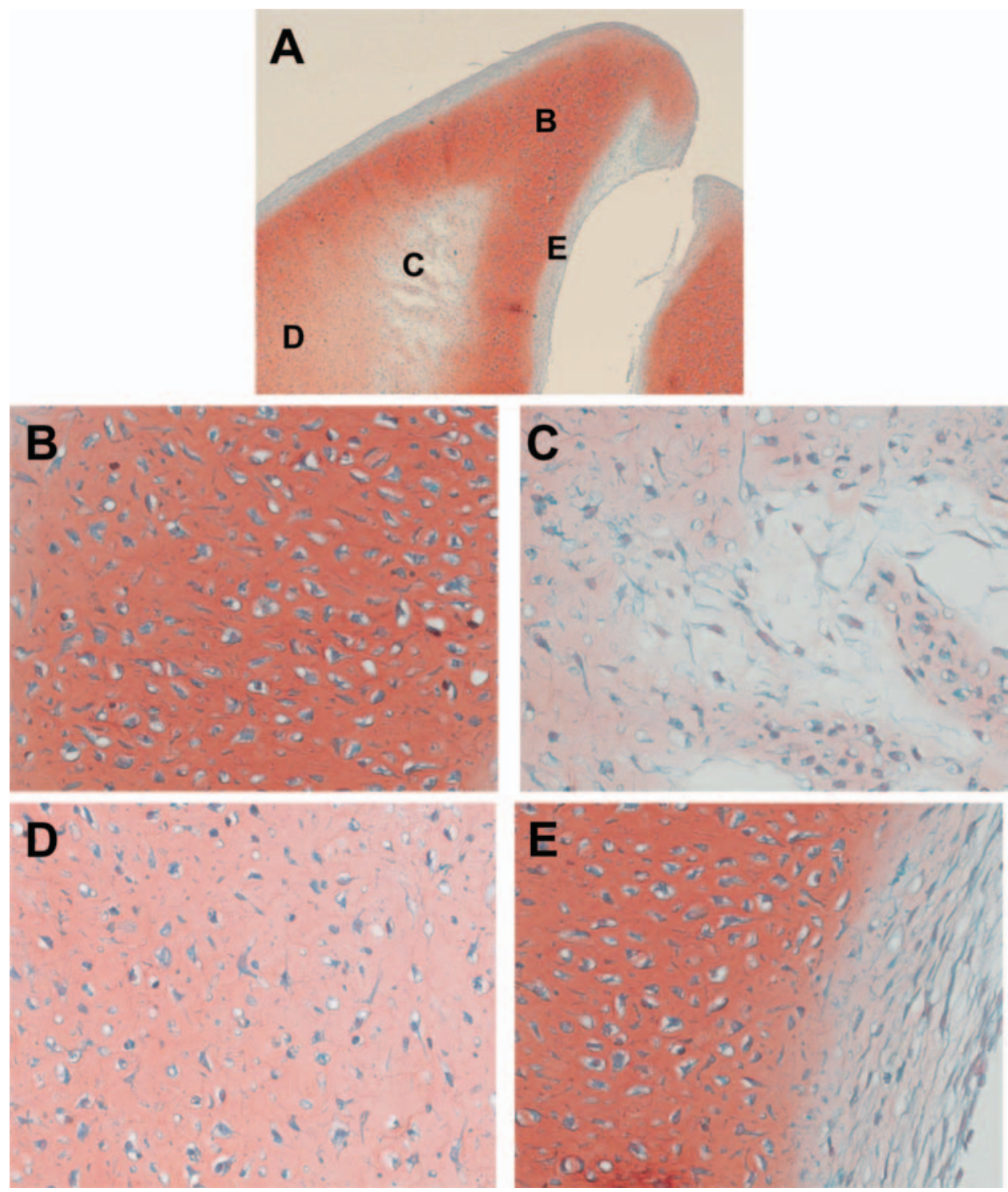

FIG. 3. Non-homogenous construct stained with Safranin O-fast green. Four regions are represented in this sample. (A) Overview with the distinct regions indicated. The rounded morphology, intensive stain, and cellular distance in (B) is approximately $50 \%$ (Grade 9), the morphology and staining intensity in (C) is approximately $20 \%$ (Grade 6), the reduced stained region located in (D) consists of approximately 20\% (Grade 8), and the low stained and fibrous tissue in (E) is approximately 10\% of the whole construct (Grade 4). Thus, this construct could be graded from 4 to 9 depending on where one chooses to grade. To score giving weight to the proportion, the scores are altered by the percentage area represented (i.e., $(9 \times 0.5+6 \times 0.2+8 \times 0.2+4 \times 0.1)=7.7)$. Recording the presence of the fibrous tissue may be important when considering the use of such material for in vivo cartilage repair (magnifications: $\mathrm{A}=10 \times$; $\mathrm{B}-\mathrm{E}=40 \times)$. Color images available online at www.liebertpub.com/ten.

type II immunohistochemistry and Alcian blue staining were considered, but to keep the approach as simple as possible, Safranin O-fast green was selected because of its previously demonstrated correlation with other methods. ${ }^{25-27}$ Alcian blue has been reported to be a less reliable means of assessing proteoglycan content, producing variability in staining intensities and localities that cannot be entirely explained. ${ }^{19}$ Although collagen type II is widely recognized as a definitive attribute of cartilage, pellet cultures and most engineered cartilages are immature and have low collagen contents, ${ }^{37,39}$ at least at the protein level at this stage, and thus GAG (a dominant molecule in such tissue) is perhaps more appropriate. Furthermore, reproducible immunolocalization of collagens is not a straightforward approach and is more expensive than most standard histological staining protocols. If the engineered constructs have been matured in culture or have been mechanically conditioned, then collagen quantifications may be of interest. Sirus Red may be a stain to consider and include in the total score because it has been shown to 

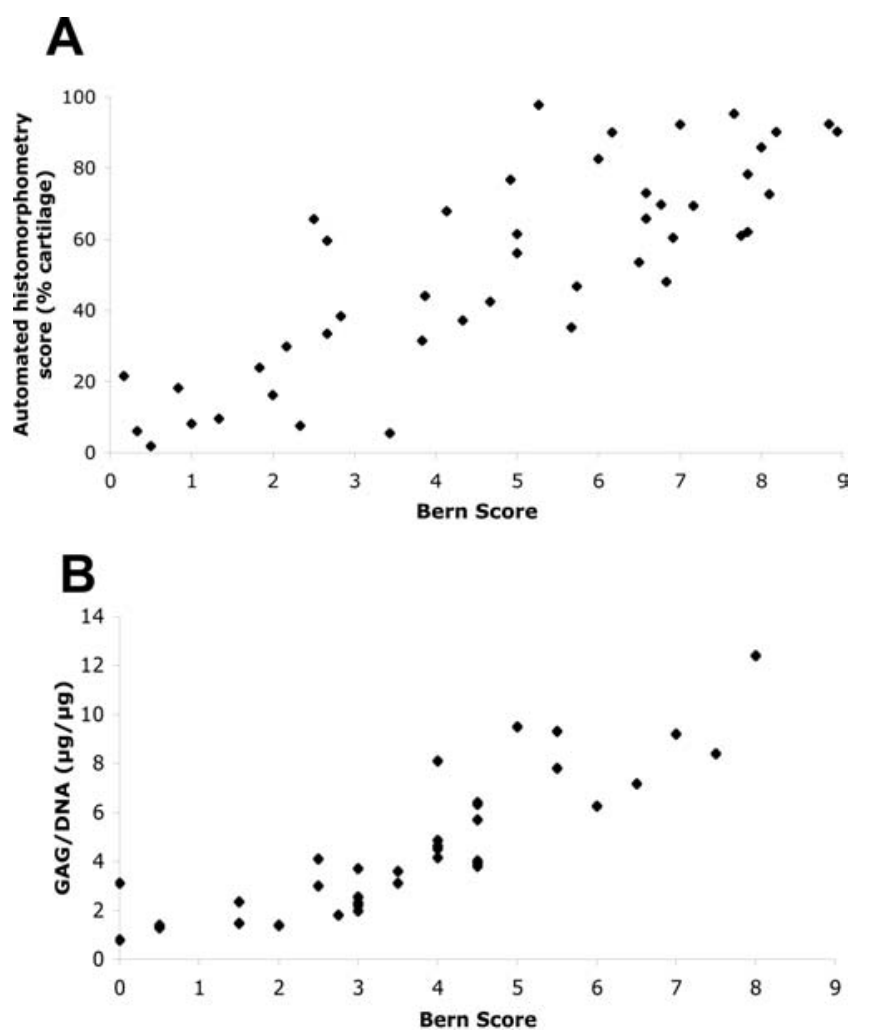

FIG. 4. (A) Relationship between Bern Score and the histomorphometric system at the Mayo Clinic $\left(r^{2}=+0.68\right.$; $p<0.0001)$. A combination of pellets $(n=25)$ and tissueengineered tissue $(n=20)$ is depicted. (B) Relationship between Bern Score and the glycosaminoglycan/deoxyribonucleic acid content in pellets $\left(r^{2}=+0.78 ; p<0.001 ; n=40\right)$.

allow differentiation between collagen subtypes when observed under polarized light. ${ }^{40}$ Nevertheless, the Bern Score may be interpreted as a "modular" system that could allow for other criteria to be added to its existing framework.

Although all tissues in this study were produced without the means of scaffolds, we believe that the Bern Score would be valid in systems that employ them. The cellular and matrix components of any cartilaginous-engineered constructs should be the main focus on the assessment of tissue quality. However, if there were particular reactions of the

Table 3. Bern Score InTra- And Inter-ObServer REPRODUCIBILITY

\begin{tabular}{lcclcc}
\hline & \multicolumn{2}{c}{$\begin{array}{c}\text { Intra-observer } \\
\text { reproducibility }\end{array}$} & & \multicolumn{2}{c}{$\begin{array}{c}\text { Inter-observer } \\
\text { reproducibility }\end{array}$} \\
\cline { 2 - 3 } \cline { 5 - 6 } Observer & Average Diff & $S D$ & & Average Diff & $S D$ \\
\hline A & 0.29 & 1.45 & & 0.1 & 0.26 \\
B & -0.33 & 1.17 & & -0.17 & 0.68 \\
C & -0.14 & 0.52 & & -0.12 & 0.49 \\
Overall & $-\mathbf{0 . 0 6}$ & $\mathbf{1 . 0 5}$ & & $-\mathbf{0 . 0 6}$ & $\mathbf{0 . 1 7}$ \\
\hline
\end{tabular}

cells to a given scaffold, the Bern Score may be a means to record these in terms of overall neo-tissue quality, the amount of matrix produced, the GAG content, and the resulting cellular morphologies represented.

\section{CONCLUSION}

Here we provide a visual scoring system that will be helpful in guiding other researchers who also need to assess chondrogenic environments. Although this system is subjective in nature, it correlated with 2 other objective parameters (biochemical and an automated computer-based histomorphometric system). The Bern Score provides an account of cell distribution and resident cell morphologies that is as descriptive as that of the established methods. Moreover, this method might be appropriate in settings that do not have access to sophisticated histomorphometric systems. Future efforts will aim at establishing whether particular scores and morphological features in engineered cartilaginous tissue may relate to the in vivo capacity to support successful repair of an articular defect.

\section{ACKNOWLEDGMENTS}

We wish to thank Mrs. Brigitte Tenisch and Mrs. Eliane Blank for their help in the histological processing. This project was supported by the Swiss National Science Foundation (SNF:4046-58623).

\section{REFERENCES}

1. Bentley, G., and Minas, T. Treating joint damage in young people. BMJ 320, 1585, 2000.

2. Lindahl, A., Brittberg, M., and Peterson, L. Cartilage repair with chondrocytes: clinical and cellular aspects. Novartis Found Symp 249, 175, 2003. Discussion 186, 234, 239.

3. Henderson, I., Tuy, B., and Oakes, B. Reoperation after autologous chondrocyte implantation. Indications and findings. J Bone Joint Surg Br 86, 205, 2004.

4. Vacanti, C.A., Kim, W., Schloo, B., Upton, J., and Vacanti, J.P. Joint resurfacing with cartilage grown in situ from cellpolymer structures. Am J Sports Med 22, 485, 1994.

5. Puelacher, W.C., Wisser, J., Vacanti, C.A., Ferraro, N.F., Jaramillo, D., and Vacanti, J.P. Temporomandibular joint disc replacement made by tissue-engineered growth of cartilage. J Oral Maxillofac Surg 52, 1172, 1994.

6. Kreklau, B., Sittinger, M., Mensing, M.B., Voigt, C., Berger, G., Burmester, G.R., Rahmanzadeh, R., and Gross, U. Tissue engineering of biphasic joint cartilage transplants. Biomaterials 20, 1743, 1999.

7. Schreiber, R.E., Ilten-Kirby, B.M., Dunkelman, N.S., Symons, K.T., Rekettye, L.M., Willoughby, J., and Ratcliffe, A. Repair of osteochondral defects with allogeneic tissue 
engineered cartilage implants. Clin Orthop 367 Suppl, S382, 1999.

8. Mainil-Varlet, P., Rieser, F., Grogan, S., Mueller, W., Saager, C., and Jakob, R.P. Articular cartilage repair using a tissueengineered cartilage-like implant: an animal study. Osteoarthr Cartil 9 Suppl A, S6, 2001.

9. Schaefer, D., Martin, I., Jundt, G., Seidel, J., Heberer, M., Grodzinsky, A., Bergin, I., Vunjak-Novakovic, G., and Freed, L.E. Tissue-engineered composites for the repair of large osteochondral defects. Arthritis Rheum 46, 2524, 2002.

10. Cancedda, R., Dozin, B., Giannoni, P., and Quarto, R. Tissue engineering and cell therapy of cartilage and bone. Matrix Biol 22, 81, 2003.

11. O'Driscoll, S.W., Keeley, F.W., and Salter, R.B. The chondrogenic potential of free autogenous periosteal grafts for biological resurfacing of major full-thickness defects in joint surfaces under the influence of continuous passive motion. An experimental investigation in the rabbit. J Bone Joint Surg Am 68, 1017, 1986.

12. Pineda, S., Pollack, A., Stevenson, S., Goldberg, V., and Caplan, A. A semiquantitative scale for histologic grading of articular cartilage repair. Acta Anat (Basel) 143, 335, 1992.

13. Mainil-Varlet, P., Aigner, T., Brittberg, M., Bullough, P., Hollander, A., Hunziker, E., Kandel, R., Nehrer, S., Pritzker, K., Roberts, S., and Stauffer, E. International Cartilage Repair Society. Histological assessment of cartilage repair: a report by the Histology Endpoint Committee of the International Cartilage Repair Society (ICRS). J Bone Joint Surg Am 85-A Suppl 2, 45, 2003.

14. Yoo, J.U., and Johnstone, B. The role of osteochondral progenitor cells in fracture repair. Clin Orthop 355 Suppl, S73, 1998.

15. Lee, G.M., Tioran, M.E., Jansen, M., Graff, R.D., Kelley, S.S., and Lin, P. Development of selective tolerance to interleukin-1beta by human chondrocytes in vitro. J Cell Physiol 192, 113, 2002.

16. Jakob, M., Demarteau, O., Schafer, D., Hintermann, B., Dick, W., Heberer, M., and Martin, I. Specific growth factors during the expansion and redifferentiation of adult human articular chondrocytes enhance chondrogenesis and cartilaginous tissue formation in vitro. J Cell Biochem 81, 368, 2001.

17. Tallheden, T., Dennis, J.E., Lennon, D.P., Sjogren-Jansson, E., Caplan, A.I., and Lindahl, A. Phenotypic plasticity of human articular chondrocytes. J Bone Joint Surg Am 85-A Suppl 2, 93, 2003.

18. Muraglia, A., Corsi, A., Riminucci, M., Mastrogiacomo, M., Cancedda, R., Bianco, P., and Quarto, R. Formation of a chondro-osseous rudiment in micromass cultures of human bone-marrow stromal cells. J Cell Sci 116, 2949, 2003.

19. Hyllested, J.L., Veje, K., and Ostergaard, K. Histochemical studies of the extracellular matrix of human articular cartilage-a review. Osteoarthr Cartil 10, 333, 2002.

20. Tay, A.G., Farhadi, J., Suetterlin, R., Pierer, G., Heberer, M., and Martin, I. Cell yield, proliferation, and postexpansion differentiation capacity of human ear, nasal, and rib chondrocytes. Tissue Eng 10, 762, 2004.

21. Mankin, H.J., Dorfman, H., Lippiello, L., and Zarins, A. Biochemical and metabolic abnormalities in articular cartilage from osteo-arthritic human hips. II. Correlation of mor- phology with biochemical and metabolic data. J Bone Joint Surg Am 53, 523, 1971.

22. Sauren, Y.M., Mieremet, R.H., Lafeber, F.P., Huber-Bruning, O., Groot, C.G., and Scherft, J.P. Changes in proteoglycans of ageing and osteoarthritic human articular cartilage: an electron microscopic study with polyethyleneimine. Anat Rec 240, 208, 1994.

23. Aigner, T., and McKenna, L. Molecular pathology and pathobiology of osteoarthritic cartilage. Cell Mol Life Sci 59, 5 , 2002.

24. Barbero, A., Grogan, S., Schäfer, D., Heberer, M., MainilVarlet, P., and Martin, I. Age related changes in human articular chondrocyte yield, proliferation and post-expansion chondrogenic capacity. Osteoarthr Cartil 12, 476, 2004.

25. Jubb, R.W., and Eggert, F.M. Staining of demineralized cartilage. II. Quantitation of articular cartilage proteoglycan after fixation and rapid demineralization. Histochemistry 73, 391, 1981.

26. Kiviranta, I., Jurvelin, J., Tammi, M., Saamanen, A.M., and Helminen, H.J. Microspectrophotometric quantitation of glycosaminoglycans in articular cartilage sections stained with Safranin O. Histochemistry 82, 249, 1985.

27. Kiraly, K., Lapvetelainen, T., Arokoski, J., Torronen, K., Modis, L., Kiviranta, I., and Helminen, H.J. Application of selected cationic dyes for the semiquantitative estimation of glycosaminoglycans in histological sections of articular cartilage by microspectrophotometry. Histochem J 28, 577, 1996.

28. Puustjarvi, K., Lammi, M., Kiviranta, I., Helminen, H.J., and Tammi, M. Proteoglycan synthesis in canine intervertebral discs after long-distance running training. J Orthop Res 11, 738, 1993.

29. O'Driscoll, S.W., Recklies, A.D., and Poole, A.R. Chondrogenesis in periosteal explants. An organ culture model for in vitro study. J Bone Joint Surg Am 76, 1042, 1994.

30. Howard, R.D., McIlwraith, C.W., Trotter, G.W., Powers, B.E., McFadden, P.R., Harwood, F.L., and Amiel, D. Longterm fate and effects of exercise on sternal cartilage autografts used for repair of large osteochondral defects in horses. Am J Vet Res 55, 1158, 1994.

31. Shimizu, C., Coutts, R.D., Healey, R.M., Kubo, T., Hirasawa, Y., and Amiel, D. Method of histomorphometric assessment of glycosaminoglycans in articular cartilage. J Orthop Res 15, 670, 1997.

32. Martin, I., Obradovic, B., Freed, L.E., and Vunjak-Novakovic, G. Method for quantitative analysis of glycosaminoglycan distribution in cultured natural and engineered cartilage. Ann Biomed Eng 27, 656, 1999.

33. O'Driscoll, S.W., Marx, R.G., Beaton, D.E., Miura, Y., Gallay, S.H., and Fitzsimmons, J.S. Validation of a simple histological-histochemical cartilage scoring system. Tissue Eng 7, 313, 2001.

34. O'Driscoll, S.W., Marx, R.G., Fitzsimmons, J.S., and Beaton, D.E. Method for automated cartilage histomorphometry. Tissue Eng 5, 13, 1999.

35. Grogan, S.P., Rieser, F., Winkelmann, V., Berardi, S., and Mainil-Varlet, P. A static, closed and scaffold-free bioreactor system that permits chondrogenesis in vitro. Osteoarthr Cartil 11, 403, 2003.

36. Hollander, A.P., Heathfield, T.F., Webber, C., Iwata, Y., Bourne, R., Rorabeck, C., and Poole, A.R. Increased 
damage to type II collagen in osteoarthritic articular cartilage detected by a new immunoassay. J Clin Invest 93, 1722, 1994.

37. Farndale, R.W., Buttle, D.J., and Barrett, A.J. Improved quantitation and discrimination of sulphated glycosaminoglycans by use of dimethylmethylene blue. Biochim Biophys Acta 883, 173, 1986.

38. Moojen, D.J., Saris, D.B., Auw Yang, K.G., Dhert, W.J., and Verbout, A.J. The correlation and reproducibility of histological scoring systems in cartilage repair. Tissue Eng 8, 627, 2002.

39. Fedewa, M.M., Oegema, T.R., Jr., Schwartz, M.H., MacLeod, A., and Lewis, J.L. Chondrocytes in culture produce a mechanically functional tissue. J Orthop Res 16, 227, 1998.
40. Junqueira, L.C., Cossermelli, W., and Brentani, R. Differential staining of collagens type I, II and III by Sirius Red and polarization microscopy. Arch Histol Jpn 41, 267, 1978.

Address reprint requests to: Pierre Mainil-Varlet, M.D., Ph.D. Osteoarticular Research Group Institute of Pathology University of Bern Murtenstrasse 31 3010, Bern, Switzerland

E-mail: pierre.mainil@pathology.unibe.ch 
